\title{
The existence of ground state solution to elliptic equation with exponential growth on complete noncompact Riemannian manifold
}

Chungen $\mathrm{Liu}^{1 *}$ and Yanjun $\mathrm{Liu}^{2}$

"Correspondence:

liucg@nankai.edu.cn

'School of Mathematics and

Information Science, Guangzhou

University, Guangzhou, P.R. China

Full list of author information is

available at the end of the article

\section{를 Springer}

\section{Abstract}

In this paper, we consider the following elliptic problem:

$$
-\operatorname{div}_{g}\left(\left|\nabla_{g} u\right|^{N-2} \nabla_{g} u\right)+V(x)|u|^{N-2} u=\frac{f(x, u)}{a(x)} \text { in } M,
$$

where $(M, g)$ be a complete noncompact $N$-dimensional Riemannian manifold with negative curvature, $N \geq 2, V$ is a continuous function satisfying $V(x) \geq V_{0}>0$, $a$ is a nonnegative function and $f(x, t)$ has exponential growth with $t$ in view of the Trudinger-Moser inequality. By proving some estimates together with the variational techniques, we get a ground state solution of $\left(P_{a}\right)$. Moreover, we also get a nontrivial weak solution to the perturbation problem.

MSC: $35 \mathrm{~J} 60 ; 35 \mathrm{~B} 33 ; 46 \mathrm{E} 30$

Keywords: Trudinger-Moser inequality; Riemannian manifold; Exponential growth; The ground state solution

\section{Introduction and main results}

Suppose $\Omega \subset \mathbb{R}^{N}$ is a bounded smooth domain, and we consider the following problem:

$$
-\operatorname{div}\left(|\nabla u|^{p-2} \nabla u\right)=f(x, u), \quad x \in \Omega,
$$

when $p=2,|f(x, u)| \leq c\left(|u|+|u|^{q-1}\right), 1<q \leq 2^{*}=\frac{2 N}{N-2}, N>2$, for corresponding results one may refer to Brézis [1], Brézis and Nirenberg [2], Bartsch and Willem [3], Capozzi, Fortunato and Palmieri [4]. Garcia and Alonso [5] generalized Brézis and Nirenberg's existence and nonexistence results to the $p$-Laplace equation. When $p=N$ and $f(x, u)$ behaves like $e^{\alpha|u|^{N-1}}$ as $|u| \rightarrow \infty$, problem (1.1) was studied by Adimurthi [6], Adimurthi and Yadava [7], Ruf et al. [8, 9], do Ó [10], Panda [11] and the references therein. Specially, Lam and Lu [12] study the case which does not satisfy the Ambrosetti-Rabinowitz condition. All these results are based on the Trudinger-Moser inequality $[13,14]$ and critical point theory.

(c) The Author(s) 2020. This article is licensed under a Creative Commons Attribution 4.0 International License, which permits use, sharing, adaptation, distribution and reproduction in any medium or format, as long as you give appropriate credit to the original author(s) and the source, provide a link to the Creative Commons licence, and indicate if changes were made. The images or other third party material in this article are included in the article's Creative Commons licence, unless indicated otherwise in a credit line to the material. If material is not included in the article's Creative Commons licence and your intended use is not permitted by statutory regulation or exceeds the permitted use, you will need to obtain permission directly from the copyright holder. To view a copy of this licence, visit http://creativecommons.org/licenses/by/4.0/. 
For the following semilinear Schrödinger equation:

$$
-\operatorname{div}\left(|\nabla u|^{p-2} \nabla u\right)+V(x)|u|^{p-2} u=f(x, u), \quad x \in \mathbb{R}^{N},
$$

when $p=2,|f(x, u)| \leq c\left(|u|+|u|^{q-1}\right), 1<q \leq 2^{*}=\frac{2 N}{N-2}, N>2$, for the relevant literature one is referred to Kryszewski and Szulkin [15], Alama and Li [16], Ding and Ni [17] and Jeanjean [18]. When $p=N, f(x, s)$ is continuous in $\mathbb{R}^{N} \times \mathbb{R}$ and behaves like $e^{\alpha|s|^{N-1}}$ as $|s| \rightarrow \infty$, it was studied by Cao [19] in the case $N=2$, by Panda [20], do Ó [21], Alves, Figueiredo [22] and do Ó et al. [23] in general dimensional cases. We note that the Sobolev embedding theorem can be applied to the $p<N$ case, while the Trudinger-Moser type embedding theorem can be applied to the $p=N$ case.

The Trudinger-Moser inequalities have been generalized to some Riemannian manifolds. In the case of compact Riemannian manifolds, the study of Trudinger-Moser inequalities can be traced back to Aubin [24], Cherrier [25, 26], and Fontana [27]. On a compact Riemannian surface, Ding et al. [28] established a Trudinger-Moser inequality and used it to deal with the prescribed Gaussian curvature problem by the method of blowup analysis. Motivated by the ideas of Ding et al., the existence of extremal functions was proved by Li in [29, 30], Yang also got extremal functions for several Trudinger-Moser type inequalities in [31,32].

When $(M, g)$ is any complete noncompact $N$-dimensional Riemannian manifold with no boundary, $N \geq 2$, if its Ricci curvature is bounded from below and injectivity radius is bounded from below by a positive number, Yang in [33] established some TrudingerMoser inequalities and applied them to some quasilinear equations. Recently, Yang et al. [34] derived a sharp Trudinger-Moser inequality on a complete, simply connected $N$ dimensional Riemannian manifold with negative curvature. We state it below for further use.

Proposition A ([34]) Let $(M, g)$ be a complete, simply connected $N$-dimensional Riemannian manifold with negative curvature, $N \geq 2$. Then for any $\tau>0$ there exists a positive constant $C=C(\tau, N, M)$ such that

$$
\sup _{u \in W^{1, N}(M),\|u\|_{1, \tau} \leq 1} \int_{M}\left(\Phi\left(\alpha_{N}\left|u_{n}\right|^{N-1}\right)\right) d v_{g} \leq C .
$$

Furthermore, the constant $\alpha_{N}=N \omega_{N-1}^{1 /(N-1)}$ is sharp, where $\omega_{N-1}$ is the surface measure of the unit sphere in $\mathbb{R}^{N}$. $\Phi(x)=e^{x}-\sum_{k=0}^{N-2} \frac{x^{k}}{k !}$ and

$$
\|u\|_{1, \tau}=\left(\int_{M}\left|\nabla_{g} u\right|^{N} d v_{g}+\tau \int_{M}|u|^{N} d v_{g}\right)^{\frac{1}{N}}
$$

More recently, Kristály [35] studied some geometric features of Trudinger-Moser inequalities on complete noncompact Riemannian manifolds and proved the existence of a non-zero isometry-invariant solution for a class of $N$-Laplacian equation by combining variational techniques and the symmetrization-compactness principle.

In this paper, we always assume that $(M, g)$ is a complete, simply connected $N$ dimensional Riemannian manifold with negative curvature, $N \geq 2$. And on $(M, g)$, we 
consider the following quasilinear equations:

$$
\begin{aligned}
& -\operatorname{div}_{g}\left(\left|\nabla_{g} u\right|^{N-2} \nabla_{g} u\right)+V(x)|u|^{N-2} u=\frac{f(x, u)}{a(x)} \quad \text { in } M, \\
& -\operatorname{div}_{g}\left(\left|\nabla_{g} u\right|^{N-2} \nabla_{g} u\right)+V(x)|u|^{N-2} u=\frac{f(x, u)}{a(x)}+\epsilon h(x) \text { in } M,
\end{aligned}
$$

where $\epsilon>0, h \in E^{*}, h \geq 0$ and $h \not \equiv 0,(M, g)$ is a complete noncompact $N$-dimensional Riemannian manifold with negative curvature, $N \geq 2, \nabla_{g}$ denotes its covariant derivative (the Levi-Civita connection), and $\mathrm{di}_{\mathrm{g}}$ denotes its divergence operator, $V$ is a continuous function satisfying $V(x) \geq V_{0}>0, a$ is a nonnegative function and $f(x, t)$ has exponential growth with $t$ in view of the Trudinger-Moser inequality. Since we are concerned with nonnegative weak solutions, we require that $f(x, t)=0$ for all $(x, t) \in M \times(-\infty, 0]$. Furthermore, let $O$ be a fixed point of $M$ and $d_{g}(\cdot, \cdot)$ be the geodesic distance between two points of $(M, g)$. We assume the function $a$ satisfies:

$\left(a_{1}\right) a^{-1} \in L_{\text {loc }}^{s}(M)$ for some $s>1$, i.e., $a^{-1} \in L^{s}\left(B_{\rho}(O)\right)$ for any $\rho>0$.

$\left(a_{2}\right) a^{-1}$ is bounded in $M \backslash B_{\rho}(O)$ for any $\rho>0$.

We assume the following conditions on $V$.

$\left(V_{1}\right)$ There exists a constant $V_{0}>0$ such that $V(x) \geq V_{0}$ for all $x \in M$.

$\left(V_{2}\right) \quad V(x) \rightarrow+\infty$ as $d_{g}(O, x) \rightarrow+\infty$.

The function $f$ satisfies:

$\left(f_{1}\right)$ There exist constants $\alpha_{0}, c_{1}, c_{2}>0$ such that, for all $(x, t) \in M \times \mathbb{R}^{+}$,

$$
f(x, t) \leq c_{1}|t|^{N-1}+c_{2} \Phi\left(\alpha_{0}\left|u_{n}\right|^{N-1}\right)
$$

where

$$
\Phi(x)=e^{x}-\sum_{k=0}^{N-2} \frac{x^{k}}{k !} .
$$

$\left(f_{2}\right) \lim _{|t| \rightarrow+\infty} \frac{F(x, t)}{\left.|t|\right|^{N}}=\infty$ uniformly on $x \in M$, where $F(x, t)=\int_{0}^{t} f(x, s) d s$.

$\left(f_{3}\right)$ There exists $C>0$ such that, for all $(x, t) \in M \times \mathbb{R}^{+}, F(x, t) \leq C|t|^{N}+C f(x, t)$.

Define a function space $E$ as

$$
E=\left\{u \in W^{1, N}(M): \int_{M}\left(\left|\nabla_{g} u\right|^{N}+V(x)|u|^{N}\right) d v_{g}<\infty\right\}
$$

equipped with the norm

$$
\|u\|_{E}=\left(\int_{M}\left(\left|\nabla_{g} u\right|^{N}+V(x)|u|^{N}\right) d v_{g}\right)^{\frac{1}{N}}
$$

then the assumption $V(x) \geq V_{0}>0$ implies that $E$ is a reflexive Banach space. For any $p \geq N$, we define

$$
S_{p}=\inf _{u \in E \backslash\{0\}} \frac{\|u\|_{E}}{\left(\int_{M} \frac{|u|^{p}}{a(x)} d v_{g}\right)^{\frac{1}{p}}}
$$


and

$$
\lambda_{a}=\inf _{u \in E \backslash\{0\}} \frac{\|u\|_{E}^{N}}{\int_{\mathbb{R}^{N}} \frac{|u|^{N}}{a(x)} d x} .
$$

The continuous embedding of $E \hookrightarrow W^{1, N}(M) \hookrightarrow L^{p}(M)(p \geq N)$ and the Hölder inequality imply

$$
\begin{aligned}
\int_{M} \frac{|u|^{p}}{a(x)} d v_{g} & =\int_{M \backslash B_{\rho}(O)} \frac{|u|^{p}}{a(x)} d v_{g}+\int_{B_{\rho}(O)} \frac{|u|^{p}}{a(x)} d v_{g} \\
& \leq C \int_{M \backslash B_{\rho}(O)}|u|^{p} d v_{g}+\left(\int_{B_{\rho}(O)}|u|^{p s^{\prime}} d v_{g}\right)^{\frac{1}{s^{\prime}}}\left(\int_{B_{\rho}(O)}\left(\frac{1}{a(x)}\right)^{s} d v_{g}\right)^{\frac{1}{s}} \\
& \leq C\|u\|_{E}^{p},
\end{aligned}
$$

here $s$ is stated in the condition $\left(a_{1}\right)$. Thus we have $S_{p}>0$. We now introduce the following three conditions.

$\left(f_{4}\right) \limsup \operatorname{su}_{t \rightarrow 0^{+}} \frac{N F(x, t)}{|t|^{N}}<\lambda_{a}$ uniformly in $M$.

$\left(f_{5}\right)$ There exist constants $p>N$ and $C_{p}$ such that

$$
f(x, t) \geq C_{p} t^{p-1}
$$

where

$$
C_{p}>\left(\frac{p-N}{p}\right)^{\frac{p-N}{N}}\left(\frac{s \alpha_{0}}{(s-1) \alpha_{N}}\right)^{\frac{(p-N)(N-1)}{N}} S_{p}^{p} .
$$

$\left(f_{6}\right) H(x, t)=t f(x, t)-N F(x, t)$ is strictly increasing in $t>0$.

Our main results can be stated as follows.

Theorem 1.1 Suppose $V$ satisfies $\left(V_{1}\right)$ and $\left(V_{2}\right)$, a satisfies $\left(a_{1}\right)$ and $\left(a_{2}\right), f$ satisfies $\left(f_{1}\right)-$ $\left(f_{6}\right)$. Then the problem $\left(P_{a}\right)$ has a positive ground state solution.

\section{Remark 1.2}

(1) When $(M, g)$ is the standard Euclidean space $\mathbb{R}^{\mathbb{N}}$ and $a(x)=|x|^{\eta}(0 \leq \eta<N)$, the equation $\left(P_{a}\right)$ and the perturbation problem $(P)$ have been studied by many authors such as Adimurthi and Yang [36], Yang [37], Lam and Lu [38].

(2) Comparing with the existing literature, we firstly study the ground state solution of problem $\left(P_{a}\right)$ on complete noncompact Riemannian manifold without the Ambrosetti-Rabinowitz condition.

Theorem 1.3 Suppose $V$ satisfies $\left(V_{1}\right)$ and $\left(V_{2}\right)$, a satisfies $\left(a_{1}\right)$ and $\left(a_{2}\right), f$ satisfies $\left(f_{1}\right)-\left(f_{4}\right)$ and $\left(f_{6}\right)$. Then the perturbation problem $(P)$ has a nontrivial weak solution of mountainpass type.

This paper is organized as follows: In Sect. 2, some preliminary results are introduced. In Sect. 3, we study the functionals and related compact analysis. In Sect. 4, we give a proof of Theorem 1.1. Finally, in Sect. 5, we prove Theorem 1.3. 


\section{Preliminaries}

In this section, we give some preliminaries for later use.

Lemma 2.1 Suppose a satisfies $\left(a_{1}\right)$ and $\left(a_{2}\right), V$ satisfies condition $\left(V_{1}\right)$. Let $\alpha>0$ and $\left\{u_{n}\right\}$ be a sequence satisfying

$$
\limsup _{n \rightarrow \infty}\left\|u_{n}\right\|_{E}^{\frac{N}{N-1}}<\frac{\alpha_{N}}{\alpha}
$$

where $\alpha_{N}=N \omega_{N-1}^{1 /(N-1)}$ is the sharp constant in Proposition A. Then there exist constants $t>1, C>0$, independent of $n$, such that

$$
\int_{M} \frac{\left[\Phi\left(\alpha\left|u_{n}\right|^{\frac{N}{N-1}}\right)\right]^{t}}{a(x)} d v_{g} \leq C
$$

Proof Since

$$
\limsup _{n \rightarrow \infty}\left\|u_{n}\right\|_{E}<\left(\frac{\alpha_{N}}{\alpha}\right)^{\frac{N-1}{N}}
$$

passing to a subsequence, there exists $n_{0} \in \mathbb{N}$ such that

$$
\left\|u_{n}\right\|_{E}^{\frac{N}{N-1}} \leq m<\frac{\alpha_{N}}{\alpha}, \quad \forall n \geq n_{0} .
$$

Choose $k>t s^{\prime}>1$ such that $k \alpha m<\alpha_{N}$ and $t<s$, here $s$ is stated in the condition $\left(a_{1}\right)$ and $\frac{1}{s}+\frac{1}{s^{\prime}}=1$. Now for each $n \geq n_{0}$, combining with $\frac{m}{\left\|u_{n}\right\|_{E}^{N-1}} \geq 1$,

$$
\begin{aligned}
& \int_{M} \frac{\left[\Phi\left(\alpha\left|u_{n}\right|^{\frac{N}{N-1}}\right)\right]^{t}}{a(x)} d v_{g} \\
& =\int_{B_{\rho}(O)} \frac{\left[\Phi\left(\alpha\left|u_{n}\right|^{\frac{N}{N-1}}\right)\right]^{t}}{a(x)} d v_{g}+\int_{M \backslash B_{\rho}(O)} \frac{\left[\Phi\left(\alpha\left|u_{n}\right|^{\frac{N}{N-1}}\right)\right]^{t}}{a(x)} d v_{g} \\
& \leq\left\|a^{-1}\right\|_{L^{s}\left(B_{\rho}(O)\right)}\left(\int_{B_{\rho}(O)}\left(\Phi\left(\alpha\left|u_{n}\right|^{\frac{N}{N-1}}\right)\right)^{t s^{\prime}} d v_{g}\right)^{\frac{1}{s^{\prime}}}+C \int_{M \backslash B_{\rho}(O)}\left(\Phi\left(\alpha\left|u_{n}\right|^{\frac{N}{N-1}}\right)\right)^{t} d v_{g} \\
& \leq\left\|a^{-1}\right\|_{L^{s}\left(B_{\rho}(O)\right)}\left(\int_{M} \Phi\left(t s^{\prime} \alpha\left|u_{n}\right|^{\frac{N}{N-1}}\right) d v_{g}\right)^{\frac{1}{s^{\prime}}}+C \int_{M} \Phi\left(t \alpha\left|u_{n}\right|^{\frac{N}{N-1}}\right) d v_{g} \\
& \leq C \int_{M} \Phi\left(k \alpha\left|u_{n}\right|^{\frac{N}{N-1}}\right) d v_{g} \leq C \int_{M} \Phi\left(k \alpha m\left(\frac{\left|u_{n}\right|}{\left\|u_{n}\right\|_{E}}\right)^{\frac{N}{N-1}}\right) d v_{g} .
\end{aligned}
$$

The property $\Phi(q)^{p} \leq \Phi(p q)$ (see [37]) has been used above. Finally, the result is derived from Proposition A by taking $\tau=V_{0}$ in condition $\left(V_{1}\right)$.

Lemma 2.2 Suppose $V$ satisfies $\left(V_{1}\right)$ and $\left(V_{2}\right)$, a satisfies $\left(a_{1}\right)$ and $\left(a_{2}\right)$, we have $E \hookrightarrow$ $L^{q}(M), q \geq N$. Moreover, this continuous embedding is compact. 
Proof From the condition $\left(V_{1}\right)$, we have $E \hookrightarrow W^{1, N}(M)$, together with the standard Sobolev embedding theorem $W^{1, N}(M) \hookrightarrow L^{q}(M)$ for any $q \geq N$, we immediately derive that $E$ can be continuously embedded into $L^{q}(M)$.

Next, for any $q \geq N$, one needs to prove that the above continuous embedding $E \hookrightarrow$ $L^{q}(M)$ is compact. Let $\left\{u_{n}\right\}$ be a sequence of functions with $\left\|u_{n}\right\|_{E}^{N} \leq C$ for some constant $C$, it suffices to prove that, up to a subsequence, $\left\{u_{n}\right\}$ strongly converges in $L^{q}(M)$. Obviously $\left\{u_{n}\right\}$ is also bounded in $W^{1, N}(M)$, thus we can assume that, for any $q \geq N$, there exists $u_{0} \in E$ such that up to a subsequence

$$
\left\{\begin{array}{l}
u_{n} \rightarrow u_{0} \quad \text { in } E \\
u_{n} \rightarrow u_{0} \quad \text { in } L^{q}\left(B_{\rho}(O)\right), \\
u_{n}(x) \rightarrow u_{0}(x) \quad \text { a.e. in } M .
\end{array}\right.
$$

Hence for any $\epsilon>0$, there exists some positive integral $n_{0}$, such that when $n>n_{0}$,

$$
\int_{B_{\rho}(O)}\left|u_{n}-u_{0}\right|^{N} d v_{g}<\frac{\epsilon}{2}
$$

On the other hand, for any $\epsilon>0$, from the condition $\left(V_{2}\right)$, we can assume $V(x)>4 C / \epsilon$ when $x \in M \backslash B_{\rho}(O)$, so

$$
\frac{4 C}{\epsilon} \int_{M \backslash B_{\rho}(O)}\left|u_{n}-u_{0}\right|^{N} d v_{g}<\int_{M \backslash B_{\rho}(O)} V\left|u_{n}-u_{0}\right|^{N} d v_{g} \leq 2 C,
$$

thus

$$
\int_{M \backslash B_{\rho}(O)}\left|u_{n}-u_{0}\right|^{N} d v_{g}<\frac{\epsilon}{2} .
$$

From (2.1) and (2.2), when $n>n_{0}$, we obtain

$$
\int_{M}\left|u_{n}-u_{0}\right|^{N} d v_{g}<\epsilon
$$

By Hölder's inequality and the continuous embedding $E \hookrightarrow L^{q}(M)(q \geq N)$, we have

$$
\begin{aligned}
\int_{M}\left|u_{n}-u_{0}\right|^{q} d v_{g} & =\int_{M}\left|u_{n}-u_{0}\right| \cdot\left|u_{n}-u_{0}\right|^{q-1} d v_{g} \\
& \leq\left(\int_{M}\left|u_{n}-u_{0}\right|^{N} d v_{g}\right)^{\frac{1}{N}}\left(\int_{M}\left|u_{n}-u_{0}\right|^{\frac{N(q-1)}{N-1}} d v_{g}\right)^{\frac{N-1}{N}} \\
& \leq C\left(\int_{M}\left|u_{n}-u_{0}\right|^{N} d v_{g}\right)^{\frac{1}{N}}
\end{aligned}
$$

Combining with (2.3), we see that $\left\{u_{n}\right\}$ strongly converges in $L^{q}(M)$.

Lemma 2.3 Suppose $V$ satisfies $\left(V_{1}\right)$, a satisfies $\left(a_{1}\right)$ and $\left(a_{2}\right)$. Let $\beta>0$ and $\|u\|_{E} \leq M$ such that $\beta M^{\frac{N}{N-1}}<\alpha_{N}$ and $q>N$, then

$$
\int_{M}|u|^{q} \frac{\Phi\left(\beta|u|^{N}\right)}{a(x)} d v_{g} \leq C(\beta, N)\|u\|_{E}^{q} .
$$


Proof On the one hand, using the Hölder inequality, we have

$$
\begin{aligned}
& \int_{B_{\rho}(O)}|u|^{q} \frac{\Phi\left(\beta|u|^{\frac{N}{N-1}}\right)}{a(x)} d v_{g} \\
& \leq\left\|a^{-1}\right\|_{L^{s}\left(B_{\rho}(O)\right)}\left(\int_{B_{\rho}(O)}|u|^{q k} d v_{g}\right)^{\frac{1}{k}}\left(\int_{M}\left(\Phi\left(\beta|u|^{\frac{N}{N-1}}\right)\right)^{t} d v_{g}\right)^{\frac{1}{t}},
\end{aligned}
$$

where $t>1$ is sufficiently close 1 such that $\beta t M^{\frac{N}{N-1}}<\alpha_{N}$ and $1 / s+1 / k+1 / t=1$.

On the other hand, we get

$$
\begin{aligned}
& \int_{M \backslash B_{\rho}(O)}|u|^{q} \frac{\Phi\left(\beta|u|^{\frac{N}{N-1}}\right)}{a(x)} d v_{g} \\
& \leq C\left(\int_{M}|u|^{q \gamma^{\prime}} d v_{g}\right)^{\frac{1}{\gamma^{\prime}}}\left(\int_{M}\left(\Phi\left(\beta|u|^{\frac{N}{N-1}}\right)\right)^{\gamma} d v_{g}\right)^{\frac{1}{\gamma}},
\end{aligned}
$$

where $\gamma>1$ is sufficiently close 1 such that $\beta \gamma M^{\frac{N}{N-1}}<\alpha_{N}, 1 / \gamma^{\prime}+1 / \gamma=1$.

From (2.4) and (2.5), by Proposition A and the continuous embedding of $E \hookrightarrow L^{q}\left(\mathbb{R}^{N}\right)$ $(q \geq N)$, we have

$$
\int_{M}|u|^{q} \frac{\Phi\left(\beta|u|^{\frac{N}{N-1}}\right)}{a(x)} d v_{g} \leq C(\beta, N)\|u\|_{E}^{q}
$$

This completes the proof.

\section{Functionals and compactness analysis}

We say that $u \in E$ is a weak solution of problem $\left(P_{a}\right)$ if, for all $\phi \in E$,

$$
\int_{M}\left(\left|\nabla_{g} u\right|^{N-2} \nabla_{g} u \nabla_{g} \phi+V(x)|u|^{N-2} u \phi\right) d v_{g}-\int_{M} \frac{f(x, u)}{a(x)} \phi d v_{g}=0 .
$$

Define the functional $I: E \rightarrow \mathbb{R}$ by

$$
I(u)=\frac{1}{N}\|u\|_{E}^{N}-\int_{M} \frac{F(x, u)}{a(x)} d v_{g}
$$

where $F(x, t)=\int_{0}^{t} f(x, s) d s$. $I$ is well defined and $I \in C^{1}(E, \mathbb{R})$ thanks to the TrudingerMoser inequality. A straightforward calculation shows that

$$
\left\langle I^{\prime}(u), \phi\right\rangle=\int_{M}\left|\nabla_{g} u\right|^{N-2} \nabla_{g} u \nabla_{g} \phi d v_{g}+\int_{M} V(x)|u|^{N-2} u \phi d v_{g}-\int_{M} \frac{f(x, u)}{a(x)} \phi d v_{g}
$$

for all $u, \phi \in E$. Hence, a critical point of $I$ defined in (3.1) is a weak solution of $\left(P_{a}\right)$.

In this paper, we will use the mountain-pass theorem for the existence of the Cerami sequence which was introduced in $[39,40]$.

Definition A Let $\left(E,\|\cdot\|_{E}\right)$ be a real Banach space with its dual space $\left(E^{*},\|\cdot\|_{E^{*}}\right)$. Suppose $I \in C^{1}(E, \mathbb{R})$. For $c \in \mathbb{R}$, we call $\left\{u_{n}\right\} \subset E$ a $(C)_{c}$ sequence of the functional $I$, if

$$
I\left(u_{n}\right) \rightarrow c \text { and }\left(1+\left\|u_{n}\right\|_{E}\right)\left\|I^{\prime}\left(u_{n}\right)\right\|_{E^{*}} \rightarrow 0 \quad \text { as } n \rightarrow \infty
$$


Proposition B Let $\left(E,\|\cdot\|_{E}\right)$ be a real Banach space, $I \in C^{1}(E, \mathbb{R}), I(0)=0$ and satisfies:

(i) There exist positive constants $\delta$ and $r$ such that

$$
I(u) \geq \delta \quad \text { for }\|u\|_{E}=r
$$

and

(ii) there exists $e \in E$ with $\|e\|_{E}>r$ such that

$$
I(e) \leq 0 .
$$

Define c by

$$
c=\inf _{\gamma \in \Gamma} \max _{t \in[0,1]} I(\gamma(t)),
$$

where

$$
\Gamma=:\{\gamma \in C([0,1]: E): \gamma(0)=0, \gamma(1)=e\} .
$$

Then I possesses a $(C)_{c}$ sequence.

Next we will check the geometry of the functional $I$.

Lemma 3.1 Assume that $\left(V_{1}\right),\left(f_{1}\right)$ and $\left(f_{4}\right)$ hold, a satisfies $\left(a_{1}\right)$ and $\left(a_{2}\right)$. Then there exist positive constants $\delta$ and $r$ such that

$$
I(u) \geq \delta \quad \text { for }\|u\|_{E}=r .
$$

Proof From $\left(f_{4}\right)$, there exist $\sigma, \epsilon>0$, such that if $|u| \leq \epsilon$,

$$
F(x, u) \leq \frac{\lambda_{a}-\sigma}{N}|u|^{N}
$$

for all $x \in M$. On the other hand, using $\left(f_{1}\right)$ for each $q>N$, we have

$$
\begin{aligned}
F(x, u) & \leq \frac{c_{1}}{N}|u|^{N}+c_{2}|u| \Phi\left(\alpha_{0}\left|u_{n}\right|^{\frac{N}{N-1}}\right) \\
& \leq C|u|^{q} \Phi\left(\alpha_{0}\left|u_{n}\right|^{\frac{N}{N-1}}\right)
\end{aligned}
$$

for $|u| \geq \epsilon$ and $x \in M$. Combining the above estimates, we obtain

$$
F(x, u) \leq \frac{\lambda_{a}-\sigma}{N}|u|^{N}+C|u|^{q} \Phi\left(\alpha_{0}\left|u_{n}\right|^{\frac{N}{N-1}}\right),
$$

for all $(x, u) \in M \times \mathbb{R}$. Fix $r>0$ small enough such that $\alpha_{0} r^{\frac{N}{N-1}}<\alpha_{N}$, then Lemma 2.3 implies

$$
\begin{aligned}
I(u) & =\frac{1}{N}\|u\|_{E}^{N}-\int_{M} \frac{F(x, u)}{a(x)} d v_{g} \\
& \geq \frac{1}{N}\|u\|_{E}^{N}-\frac{\lambda_{a}-\sigma}{N} \int_{M} \frac{|u|^{N}}{a(x)} d v_{g}-C \int_{M} \frac{|u|^{q} \Phi\left(\alpha_{0}\left|u_{n}\right|^{N-1}\right)}{a(x)} d v_{g}
\end{aligned}
$$




$$
\begin{aligned}
& \geq \frac{1}{N}\|u\|_{E}^{N}-\frac{\lambda_{a}-\sigma}{N} \int_{M} \frac{|u|^{N}}{a(x)} d v_{g}-C\|u\|_{E}^{q} \\
& \geq \frac{1}{N}\left(1-\frac{\lambda_{a}-\sigma}{\lambda_{a}}\right)\|u\|_{E}^{N}-C\|u\|_{E}^{q}=\frac{\sigma}{N \lambda_{a}}\|u\|_{E}^{N}-C\|u\|_{E}^{q},
\end{aligned}
$$

for $\|u\|_{E} \leq r$. Hence, $I$ is bounded from below for $\|u\|_{E} \leq r$. Since $\sigma>0$ and $q>N$, we may choose a small $r$ that satisfies the inequality

$$
\frac{\sigma}{N \lambda_{a}} r^{N}-C r^{q} \geq \frac{\sigma}{2 N \lambda_{a}} r^{N},
$$

and we derive that

$$
I(u) \geq \frac{\sigma}{2 N \lambda_{a}} r^{N}:=\delta>0 \quad \text { for }\|u\|_{E}=r .
$$

This completes the proof.

Lemma 3.2 If the condition $\left(f_{2}\right)$ is satisfied, then there exists $e \in B_{r}^{c}(0)$ such that

$$
I(e)<\inf _{\|u\|_{E}=r} I(u)
$$

where $r$ is given in Lemma 3.1.

Proof Let $u \in E \backslash\{0\}, u \geq 0$ with compact support $\Omega=\operatorname{supp}(u)$, by $\left(f_{2}\right)$, there exists $d$ such that, for all $(x, s) \in \Omega \times \mathbb{R}^{+}$,

$$
F(x, s) \geq M s^{N}-d .
$$

Then

$$
\begin{aligned}
I(t u) & \leq \frac{t^{N}}{N}\|u\|_{E}^{N}-M t^{N} \int_{\Omega} \frac{|u|^{N}}{a(x)} d v_{g}+O(1) \\
& \leq t^{N}\left(\frac{\|u\|_{E}^{N}}{N}-M \int_{\Omega} \frac{|u|^{N}}{a(x)} d v_{g}\right)+O(1) .
\end{aligned}
$$

Now, choose $M>\frac{\|u\|_{E}^{N}}{\int_{\Omega} \frac{\mid u^{N}}{a(x)} d v_{g}}$, which implies that $I(t u) \rightarrow-\infty$ as $t \rightarrow \infty$. Setting $e=t u$ with $t$ sufficiently large, the proof of the lemma is completed.

From Lemmas 3.1 and 3.2, we get a $(C)_{c}$ sequence $\left\{u_{n}\right\} \subset E$ by using Proposition B, i.e.,

$$
I\left(u_{n}\right) \rightarrow c>0 \quad \text { and } \quad\left(1+\left\|u_{n}\right\|_{E}\right)\left\|I^{\prime}\left(u_{n}\right)\right\|_{E^{*}} \rightarrow 0 \quad \text { as } n \rightarrow \infty
$$

where

$$
c=\inf _{\gamma \in \Gamma} \max _{t \in[0,1]} I(\gamma(t))
$$

and

$$
\Gamma=:\{\gamma \in C([0,1]: E): \gamma(0)=0, \gamma(1)=e\} .
$$


Lemma 3.3 Suppose $V$ satisfies $\left(V_{1}\right)$ and $\left(V_{2}\right)$, a satisfies $\left(a_{1}\right)$ and $\left(a_{2}\right), f$ satisfies $\left(f_{5}\right)$, then the level $c \in\left(0, \frac{1}{N}\left(\left(1-\frac{1}{s}\right) \frac{\alpha_{N}}{\alpha_{0}}\right)^{N-1}\right)$.

Proof Firstly, we claim the best constant $S_{p}(p>N)$ in (1.3) can be obtained. In fact, since

$$
S_{p}=\inf _{u \in E \backslash\{0\}} \frac{\|u\|_{E}}{\left(\int_{M} \frac{|u|^{p}}{a(x)} d v_{g}\right)^{\frac{1}{p}}},
$$

we can choose $u_{n}$ such that

$$
\int_{M} \frac{\left|u_{n}\right|^{p}}{a(x)} d v_{g}=1 \quad \text { and } \quad\left\|u_{n}\right\|_{E} \rightarrow S_{p} \quad \text { as } n \rightarrow \infty
$$

so $u_{n}$ is bounded in $E$. From Lemma 2.2, there exists $u \in E$ such that up to a subsequence $u_{n} \rightarrow u$ in $E, u_{n} \rightarrow u$ in $L^{p}(M)$ and $u_{n}(x) \rightarrow u(x)$ almost everywhere in $M$, so $u_{n} \rightarrow u$ in $L^{q}\left(B_{\rho}(O)\right)$ for all $q>1$ and $a \in L^{s}\left(B_{\rho}(O)\right)$, by the Hölder inequality

$$
\int_{B_{\rho}(O)} \frac{\left|u_{n}\right|^{p}}{a(x)} d v_{g} \rightarrow \int_{B_{\rho}(O)} \frac{|u|^{p}}{a(x)} d v_{g} .
$$

From $\left(a_{2}\right)$, we have

$$
\begin{aligned}
\int_{M \backslash B_{\rho}(O)}\left|\frac{\left|u_{n}\right|^{p}-|u|^{p}}{a(x)}\right| d v_{g} & \leq p C \int_{M}\left(\left|u_{n}\right|^{p-1}+|u|^{p-1}\right)\left|u_{n}-u\right| d v_{g} \\
& \leq C\left(\int_{M}\left|u_{n}-u\right|^{p} d v_{g}\right)^{\frac{1}{p}} \rightarrow 0 .
\end{aligned}
$$

This implies

$$
\int_{M} \frac{|u|^{p}}{a(x)} d x=\lim _{n \rightarrow \infty} \int_{M} \frac{\left|u_{n}\right|^{p}}{a(x)} d x=1 .
$$

We also have $\|u\|_{E} \leq \lim _{n \rightarrow \infty}\left\|u_{n}\right\|_{E}=S_{p}$, thus $\|u\|_{E}=S_{p}$. We finished the proof of the claim.

We claim that there exists a number $t_{0}>r$ such that for the above $u$ we have

$$
I\left(t_{0} u\right)<0,
$$

namely, we can take $e=t_{0} u$ as in Lemma 3.2. In fact, for $t>0$, by condition $\left(f_{5}\right)$, we have

$$
I(t u) \leq \frac{t^{N}}{N} S_{p}^{N}-\frac{t^{p}}{p} C_{p} .
$$

Since $p>N$, by choosing $t_{0}$ large enough we get the claim clearly.

From the definition of $c$, take $\gamma:[0,1] \rightarrow E, \gamma(t)=t t_{0} u$ with $e=t_{0} u$ as claimed above. We have $\gamma \in \Gamma$ and therefore

$$
\begin{aligned}
c & \leq \max _{t \in[0,1]} I(\gamma(t)) \leq \max _{t \geq 0} I(t u) \\
& \leq \max _{t \geq 0}\left(\frac{t^{N}}{N} S_{p}^{N}-\frac{t^{p}}{p} C_{p}\right)
\end{aligned}
$$




$$
\begin{aligned}
& =\frac{p-N}{N p} \frac{S_{p}^{\frac{N p}{p-N}}}{C_{p}^{\frac{N}{p-N}}} \\
& <\frac{1}{N}\left(\left(1-\frac{1}{s}\right) \frac{\alpha_{N}}{\alpha_{0}}\right)^{N-1} .
\end{aligned}
$$

In the last estimation we have used the inequality in condition $\left(f_{5}\right)$. The proof of Lemma 3.3 is completed.

It is well known that the absence of the Cerami compactness condition brings about difficulties in studying this class of elliptic problems involving critical growth and unbounded domains. In the next lemma, we will analyze the compactness of Cerami sequences for $I$.

Lemma 3.4 Suppose that the conditions $\left(V_{1}\right)-\left(V_{2}\right),\left(f_{1}\right)-\left(f_{4}\right)$ and $\left(f_{6}\right)$ are satisfied, a satisfies $\left(a_{1}\right)$ and $\left(a_{2}\right)$, let $\left\{u_{n}\right\} \subset E$ be an arbitrary Cerami sequence of $I$, then there exists a subsequence of $\left\{u_{n}\right\}$ (still denoted by $\left.\left\{u_{n}\right\}\right)$ and $u \in E$ such that

$$
\left\{\begin{array}{l}
\frac{f\left(x, u_{n}\right)}{a(x)} \rightarrow \frac{f(x, u)}{a(x)} \quad \text { strongly in } L_{\mathrm{loc}}^{1}(M), \\
\frac{F\left(x, u_{n}\right)}{a(x)} \rightarrow \frac{F(x, u)}{a(x)} \quad \text { strongly in } L^{1}(M), \\
\nabla_{g} u_{n} \rightarrow \nabla_{g} u \quad \text { almost everywhere in } M, \\
\left|\nabla_{g} u_{n}\right|^{N-2} \nabla_{g} u_{n} \rightarrow\left|\nabla_{g} u\right|^{N-2} \nabla_{g} u \quad \text { weakly in }\left(L^{N /(N-1)}(M)\right)^{N} .
\end{array}\right.
$$

Furthermore, $u$ is a weak solution of $\left(P_{a}\right)$.

Proof We shall prove that $\left\{u_{n}\right\}$ is bounded in $E$. Indeed, suppose by contradiction that

$$
\left\|u_{n}\right\|_{E} \rightarrow+\infty
$$

and set

$$
v_{n}=\frac{u_{n}}{\left\|u_{n}\right\|_{E}},
$$

then $\left\|v_{n}\right\|=1$. From Lemma 2.2, we can assume that, for any $q \geq N$, there exists $v \in E$ such that up to a subsequence

$$
\begin{cases}v_{n}^{+} \rightarrow v^{+} & \text {in } E, \\ v_{n}^{+} \rightarrow v^{+} & \text {in } L^{q}(M), \\ v_{n}^{+} \rightarrow v^{+} & \text {a.e. in } M .\end{cases}
$$

We will show that $v^{+}=0$ a.e. in $M$. In fact, if $\Lambda^{+}=\left\{x \in M: v^{+}(x)>0\right\}$ has a positive measure, then in $\Lambda^{+}$, we have

$$
\lim _{n \rightarrow \infty} u_{n}^{+}=\lim _{n \rightarrow \infty} v_{n}^{+}\left\|u_{n}\right\|=+\infty .
$$

From $\left(f_{2}\right)$ we have

$$
\lim _{n \rightarrow \infty} \frac{F\left(x, u_{n}^{+}(x)\right)}{a(x)\left|u_{n}^{+}(x)\right|^{N}}=+\infty \quad \text { a.e. in } \Lambda^{+}
$$


and

$$
\lim _{n \rightarrow \infty} \frac{F\left(x, u_{n}^{+}(x)\right)}{a(x)\left|u_{n}^{+}(x)\right|^{N}}\left|v_{n}^{+}(x)\right|^{N}=+\infty \quad \text { a.e. in } \Lambda^{+} .
$$

Thus

$$
\int_{M} \liminf _{n \rightarrow \infty} \frac{F\left(x, u_{n}^{+}(x)\right)}{a(x)\left|u_{n}^{+}(x)\right|^{N}}\left|v_{n}^{+}(x)\right|^{N} d v_{g}=+\infty .
$$

Since $\left\{u_{n}\right\} \subset E$ is an arbitrary Cerami sequence of $I$, we have

$$
\left\|u_{n}\right\|_{E}^{N}=N c+N \int_{M} \frac{F\left(x, u_{n}^{+}(x)\right)}{a(x)} d v_{g}+o_{n}(1)
$$

then

$$
\int_{M} \frac{F\left(x, u_{n}^{+}(x)\right)}{a(x)} d v_{g} \rightarrow+\infty
$$

SO

$$
\begin{aligned}
& \liminf _{n \rightarrow \infty} \int_{M} \frac{F\left(x, u_{n}^{+}(x)\right)}{a(x)\left|u_{n}^{+}(x)\right|^{N}}\left|v_{n}^{+}(x)\right|^{N} d v_{g} \\
& \quad=\liminf _{n \rightarrow \infty} \int_{M} \frac{F\left(x, u_{n}^{+}(x)\right)}{a(x)\left\|u_{n}\right\|^{N}} d v_{g} \\
& \quad=\liminf _{n \rightarrow \infty} \frac{\int_{M} \frac{F\left(x, u_{n}^{+}(x)\right)}{a(x)} d v_{g}}{N c+N \int_{M} \frac{F\left(x, u_{n}^{+}(x)\right)}{a(x)} d v_{g}+o_{n}(1)} \\
& \quad=\frac{1}{N} .
\end{aligned}
$$

This is a contradiction. Hence $v \leq 0$ a.e. and $v_{n}^{+} \rightarrow 0$ in $E$.

Let $t_{n} \in[0,1]$ be such that

$$
I\left(t_{n} u_{n}\right)=\max _{t \in[0,1]} I\left(t u_{n}\right)
$$

For any given $A \in\left(0,\left(\left(1-\frac{1}{s}\right) \frac{\alpha_{N}}{\alpha_{0}}\right)^{\frac{N-1}{N}}\right)$, for the sake of simplicity, let

$$
\epsilon=\frac{\left(1-\frac{1}{s}\right) \alpha_{N}}{A^{N-1}}-\alpha_{0}>0 .
$$

In the following argument we will take $A \rightarrow\left(\left(1-\frac{1}{s}\right) \frac{\alpha_{N}}{\alpha_{0}}\right)^{\frac{N-1}{N}}$ and so we have $\epsilon \rightarrow 0$. From condition $\left(f_{1}\right)$, there exists $C>0$ such that

$$
F(x, t) \leq C|t|^{N}+\epsilon \Phi\left(\left(\alpha_{0}+\epsilon\right)|t|^{\frac{N}{N-1}}\right), \quad \forall(x, t) \in M \times \mathbb{R}^{+} .
$$

In fact, from condition $\left(f_{1}\right)$, we have

$$
F(x, t) \leq \frac{C}{N}|t|^{N}+|t| \Phi\left(\alpha_{0}|t|^{\frac{N}{N-1}}\right) .
$$


By using the Young inequality, for $\frac{1}{p}+\frac{1}{q}=1, p, q>1$, we have

$$
a b \leq \epsilon \frac{a^{p}}{p}+\epsilon^{-q / p} \frac{b^{q}}{q} .
$$

So we have

$$
F(x, t) \leq \frac{C}{N}|t|^{N}+\frac{\epsilon \Phi\left(p \alpha_{0}|t| \frac{N}{N-1}\right)}{p}+\epsilon^{-q / p} \frac{|t|^{q}}{q} .
$$

Now we take $p=\frac{\alpha_{0}+\epsilon}{\alpha_{0}}$ and $q=\frac{\alpha_{0}+\epsilon}{\epsilon}>N$. One can see that near infinity $|t|^{q}$ can be estimated from above by $\Phi\left(\left(\alpha_{0}+\epsilon\right)|t|^{\frac{N}{N-1}}\right)$, and near the origin $|t|^{q}$ can be estimated from above by $|t|^{N}$.

We also have $\frac{A}{\left\|u_{n}\right\|} \in[0,1]$ with sufficient large $n$, so by using (3.4)

$$
\begin{aligned}
I\left(t_{n} u_{n}\right) & \geq I\left(\frac{A}{\left\|u_{n}\right\|} u_{n}\right)=I\left(A v_{n}\right) \\
& =\frac{A^{N}}{N}-\int_{M} \frac{F\left(x, A v_{n}\right)}{a(x)} d v_{g} \\
& =\frac{A^{N}}{N}-\int_{M} \frac{F\left(x, A v_{n}^{+}\right)}{a(x)} d v_{g} \\
& \geq \frac{A^{N}}{N}-\frac{C}{N} A^{N} \int_{M} \frac{\left|v_{n}^{+}\right|^{N}}{a(x)} d v_{g}-\epsilon \int_{M} \frac{\Phi\left(\left(\alpha_{0}+\epsilon\right)\left|A v_{n}^{+}\right| \frac{N}{N-1}\right)}{a(x)} d v_{g} \\
& \geq \frac{A^{N}}{N}-\frac{C}{N} A^{N} \int_{M} \frac{\left|v_{n}^{+}\right|^{N}}{a(x)} d v_{g}-\epsilon \int_{M} \frac{\Phi\left(\left(1-\frac{1}{s}\right) \alpha_{N}\left|v_{n}^{+}\right| \frac{N}{N-1}\right)}{a(x)} d v_{g} .
\end{aligned}
$$

Since $v_{n}^{+} \rightarrow 0$ in $E$ and the embedding $E \hookrightarrow L^{q}(M)(q \geq N)$ is compact, by using the Hölder inequality, we have $\int_{M} \frac{\left|v_{n}^{+}\right|^{N}}{a(x)} d v_{g} \rightarrow 0$. By Proposition A, $\int_{M} \frac{\Phi\left(\left(1-\frac{1}{s}\right) \alpha_{N}\left|v_{n}^{+}\right| \frac{N}{N-1}\right)}{a(x)} d v_{g}$ is bounded. Note that when $A \rightarrow\left(\frac{(s-1) \alpha_{N}}{s \alpha_{0}}\right)^{\frac{N-1}{N}}, \epsilon \rightarrow 0$, we can show

$$
\liminf _{n \rightarrow \infty} I\left(t_{n} u_{n}\right) \geq \frac{1}{N}\left(\left(1-\frac{1}{s}\right) \frac{\alpha_{N}}{\alpha_{0}}\right)^{N-1}>c .
$$

Notice $I(0)=0$ and $I\left(u_{n}\right) \rightarrow c$, we can assume $t_{n} \in(0,1)$, and so $I^{\prime}\left(t_{n} u_{n}\right) t_{n} u_{n}=0$, it follows from $\left(f_{6}\right)$ that

$$
\begin{aligned}
N I\left(t_{n} u_{n}\right) & =N I\left(t_{n} u_{n}\right)-I^{\prime}\left(t_{n} u_{n}\right) t_{n} u_{n} \\
& =\left\|t_{n} u_{n}\right\|^{N}-N \int_{M} \frac{F\left(x, t_{n} u_{n}\right)}{a(x)} d v_{g}-\left\|t_{n} u_{n}\right\|^{N}+\int_{M} \frac{f\left(x, t_{n} u_{n}\right) t_{n} u_{n}}{a(x)} d v_{g} \\
& =\int_{M} \frac{H\left(x, t_{n} u_{n}\right)}{a(x)} d v_{g} \leq \int_{M} \frac{H\left(x, u_{n}\right)}{a(x)} d v_{g} \\
& =N I\left(u_{n}\right)-I^{\prime}\left(u_{n}\right) u_{n} \\
& =N I\left(u_{n}\right)+o_{n}(1)=N c+o_{n}(1),
\end{aligned}
$$


which is a contradiction to (3.5). This proves that $\left\{u_{n}\right\}$ is bounded in $E$. It then follows from (3.3) that

$$
\int_{M} \frac{f\left(x, u_{n}\right) u_{n}}{a(x)} d v_{g} \leq C, \quad \int_{M} \frac{F\left(x, u_{n}\right)}{a(x)} d v_{g} \leq C .
$$

By $\left(f_{3}\right)$, there exists $C>0$ such that

$$
F\left(x, u_{n}\right) \leq C\left|u_{n}\right|^{N}+C f\left(x, u_{n}\right) .
$$

From Lemma 2.2 and the generalized Lebesgue dominated convergence theorem, discussed as Lemma 7.6 in [33], we can derive that

$$
\frac{f\left(x, u_{n}\right)}{a(x)} \rightarrow \frac{f(x, u)}{a(x)} \quad \text { strongly in } L_{\mathrm{loc}}^{1}(M)
$$

and

$$
\frac{F\left(x, u_{n}\right)}{a(x)} \rightarrow \frac{F(x, u)}{a(x)} \quad \text { strongly in } L^{1}(M) .
$$

Now we prove the remaining part of the lemma. Up to a subsequence, we can define an energy concentration set for any fixed $\delta>0$,

$$
\Sigma_{\delta}=\left\{x \in M: \lim _{r \rightarrow 0} \lim _{n \rightarrow \infty} \int_{\mathbb{B}_{r}(x)}\left(\left|\nabla_{g} u_{n}\right|^{N}+\left|u_{n}\right|^{N}\right) d v_{g} \geq \delta\right\} .
$$

Since $\left\{u_{n}\right\}$ is bounded in $E, \Sigma_{\delta}$ must be a finite set. For any $x^{*} \in M \backslash \Sigma_{\delta}$, there exists $r: 0<r<d_{g}\left(x^{*}, \Sigma_{\delta}\right)$ such that

$$
\lim _{n \rightarrow \infty} \int_{\mathbb{B}_{r}\left(x^{*}\right)}\left(\left|\nabla_{g} u_{n}\right|^{N}+\left|u_{n}\right|^{N}\right) d v_{g}<\delta
$$

It follows that, for large $n$,

$$
\int_{\mathbb{B}_{r}\left(x^{*}\right)}\left(\left|\nabla_{g} u_{n}\right|^{N}+\left|u_{n}\right|^{N}\right) d v_{g}<\delta
$$

Thanks to the Trudinger-Moser inequality in Lemma 2.1, for sufficiently small $\delta>0$, there exists $q>1$ such that

$$
\int_{\mathbb{B}_{r}\left(x^{*}\right)} \frac{\left|f\left(x, u_{n}\right)\right|^{q}}{a(x)} d v_{g} \leq C .
$$

For any $L>0$, we denote

$$
A_{L}=\left\{x \in \mathbb{B}_{r}\left(x^{*}\right):|u(x)| \geq L\right\} .
$$


It can be estimated that

$$
\begin{aligned}
\int_{A_{L}} & \frac{\left|f\left(x, u_{n}\right)-f(x, u)\right||u|}{a(x)} d v_{g} \\
\leq & \left(\int_{A_{L}} \frac{\left|f\left(x, u_{n}\right)-f(x, u)\right|^{q}}{a(x)} d v_{g}\right)^{\frac{1}{q}}\left(\int_{A_{L}} \frac{|u|^{q^{\prime}}}{a(x)} d v_{g}\right)^{\frac{1}{q^{\prime}}} \\
\leq & {\left[\left(\int_{\mathbb{B}_{r}\left(x^{*}\right)} \frac{\left|f\left(x, u_{n}\right)\right|^{q}}{a(x)} d v_{g}\right)^{\frac{1}{q}}+\left(\int_{\mathbb{B}_{r}\left(x^{*}\right)} \frac{|f(x, u)|^{q}}{a(x)} d v_{g}\right)^{\frac{1}{q}}\right] } \\
& \times\left(\int_{\mathbb{B}_{r}\left(x^{*}\right)} \frac{1}{a(x)^{s}} d v_{g}\right)^{\frac{1}{q^{\prime} s}}\left(\int_{A_{M}}|u|^{q^{\prime} t^{\prime}} d x\right)^{\frac{1}{q^{\prime} t^{\prime}}} \\
\leq & C\left(\int_{A_{L}}|u|^{q^{\prime} t^{\prime}} d v_{g}\right)^{\frac{1}{q^{\prime} t^{\prime}}},
\end{aligned}
$$

where $\frac{1}{q}+\frac{1}{q^{\prime}}=1, \frac{1}{s}+\frac{1}{s^{\prime}}=1$. Here we have used (3.9) in the last inequality. Since $u \in$ $L^{q^{\prime} t^{\prime}}\left(\mathbb{B}_{r}\left(x^{*}\right)\right)$, we have, for any $\beta>0$,

$$
\int_{A_{L}} \frac{\left|f\left(x, u_{n}\right)-f(x, u)\right||u|}{a(x)} d v_{g}<\beta
$$

provided that $L$ is chosen sufficiently large. It follows from (3.6) that

$$
\lim _{n \rightarrow \infty} \int_{\mathbb{B}_{r}\left(x^{*}\right) \backslash A_{L}} \frac{\left|f\left(x, u_{n}\right)-f(x, u)\right||u|}{a(x)} d v_{g}=0 .
$$

Combining (3.10) and (3.11), we have

$$
\lim _{n \rightarrow \infty} \int_{\mathbb{B}_{r}\left(x^{*}\right)} \frac{\left|f\left(x, u_{n}\right)-f(x, u)\right||u|}{a(x)} d v_{g} \leq \beta
$$

and thanks to the fact that $\beta>0$ is arbitrary,

$$
\lim _{n \rightarrow \infty} \int_{\mathbb{B}_{r}\left(x^{*}\right)} \frac{\left|f\left(x, u_{n}\right)-f(x, u)\right||u|}{a(x)} d v_{g}=0 .
$$

On the other hand, we have by using the Hölder inequality, (3.6), and (3.9),

$$
\begin{aligned}
& \int_{\mathbb{B}_{r}\left(x^{*}\right)} \frac{\left|f\left(x, u_{n}\right)\right|\left|u_{n}-u\right|}{a(x)} d v_{g} \\
& \leq\left(\int_{\mathbb{B}_{r}\left(x^{*}\right)} \frac{\left|f\left(x, u_{n}\right)\right|^{q}}{a(x)} d v_{g}\right)^{\frac{1}{q}}\left(\int_{\mathbb{B}_{r}\left(x^{*}\right)} \frac{1}{a(x)^{s}} d v_{g}\right)^{\frac{1}{q^{\prime} s}}\left\|u_{n}-u\right\|_{L^{q^{\prime} s^{\prime}}\left(\mathbb{B}_{r}\left(x^{*}\right)\right)} \\
& \leq C\left\|u_{n}-u\right\|_{L^{q^{\prime} s^{\prime}}\left(\mathbb{B}_{r}\left(x^{*}\right)\right)} \rightarrow 0,
\end{aligned}
$$

where $\frac{1}{q}+\frac{1}{q^{\prime}}=1, \frac{1}{s}+\frac{1}{s^{\prime}}=1$. Combining (3.12) and (3.13), we get

$$
\lim _{n \rightarrow \infty} \int_{\mathbb{B}_{r}\left(x^{*}\right)} \frac{\left|f\left(x, u_{n}\right)-f(x, u) u\right|}{a(x)} d v_{g}=0 .
$$


A covering argument implies that, for any compact set $K \subset \subset M \backslash \Sigma_{\delta}$,

$$
\lim _{n \rightarrow \infty} \int_{K} \frac{\left|f\left(x, u_{n}\right)-f(x, u) u\right|}{a(x)} d v_{g}=0 .
$$

Next, we will prove for any compact set $K \subset \subset M \backslash \Sigma_{\delta}$

$$
\lim _{n \rightarrow \infty} \int_{K}\left|\nabla_{g} u_{n}-\nabla_{g} u\right|^{N} d v_{g}=0
$$

It suffices to prove for any $x^{*} \in M \backslash \Sigma_{\delta}$ and $r$ given by (3.9), we have

$$
\lim _{n \rightarrow \infty} \int_{\mathbb{B}_{\frac{r}{2}\left(x^{*}\right)}}\left|\nabla_{g} u_{n}-\nabla_{g} u\right|^{N} d v_{g}=0
$$

For this purpose, we take $\phi \in C_{0}^{\infty}\left(\mathbb{B}_{r}\left(x^{*}\right)\right)$ with $0 \leq \phi \leq 1$ and $\phi \equiv 1$ in $\mathbb{B}_{\frac{r}{2}}\left(x^{*}\right)$. By the Hölder inequality and the compact embedding of $E \hookrightarrow L^{N}(M)$, we have

$$
\lim _{n \rightarrow \infty} \int_{\mathbb{B}_{r}\left(x^{*}\right)}\left|\nabla_{g} u_{n}\right|^{N-2} \nabla_{g} u_{n} \nabla_{g} \phi\left(u-u_{n}\right) d v_{g}=0 .
$$

Since $\nabla u_{n} \rightarrow \nabla u$ in $\left(L^{N}(M)\right)^{N}$, we have

$$
\lim _{n \rightarrow \infty} \int_{\mathbb{B}_{r}\left(x^{*}\right)} \phi\left|\nabla_{g} u_{n}\right|^{N-2} \nabla_{g} u\left(\nabla_{g} u-\nabla_{g} u_{n}\right) d v_{g}=0 .
$$

The Hölder inequality and (3.13) implies that

$$
\lim _{n \rightarrow \infty} \int_{\mathbb{B}_{r}\left(x^{*}\right)} \phi\left(u_{n}-u\right) \frac{f\left(x, u_{n}\right)}{a(x)} d v_{g} \rightarrow 0 .
$$

Note that

$$
\left\langle|x|^{N-2} x-|y|^{N-2} y, x-y\right\rangle=\frac{|x|^{N-2}+|y|^{N-2}}{2}|x-y|^{2}+\frac{\left(|x|^{N-2}-|y|^{N-2}\right)\left(|x|^{2}-|y|^{2}\right)}{2} .
$$

Hence,

$$
\left\langle|x|^{N-2} x-|y|^{N-2} y, x-y\left|\geq \frac{|x|^{N-2}+|y|^{N-2}}{2}\right| x-\left.y\right|^{2} \geq 2^{2-N}|x-y|^{N} .\right.
$$

Obviously $\phi u_{n}$ is a bounded sequence in $E$. Inserting $\varphi=\phi u_{n}$ and $\varphi=\phi u$ into (3.3), respectively, we have from (3.17)-(3.20) and $\tau_{n} \rightarrow 0$ as $n \rightarrow \infty$

$$
\begin{aligned}
0 \leq & 2^{2-N} \int_{\mathbb{B}_{r}\left(x^{*}\right)}\left|\nabla_{g} u_{n}-\nabla_{g} u\right|^{N} d v_{g} \\
\leq & \left.\left.\int_{\mathbb{B}_{r}\left(x^{*}\right)} \phi|| \nabla_{g} u_{n}\right|^{N-2} \nabla_{g} u_{n}-\left|\nabla_{g} u\right|^{N-2} \nabla_{g} u, \nabla_{g} u_{n}-\nabla_{g} u\right\rangle d v_{g} \\
\leq & \int_{\mathbb{B}_{r}\left(x^{*}\right)}\left|\nabla_{g} u_{n}\right|^{N-2} \nabla_{g} u_{n} \nabla_{g} \phi\left(u-u_{n}\right) d v_{g}+\int_{B_{r}\left(x^{*}\right)} \phi\left|\nabla_{g} u\right|^{N-2} \nabla_{g} u\left(\nabla_{g} u-\nabla_{g} u_{n}\right) d v_{g} \\
& +\int_{\mathbb{B}_{r}\left(x^{*}\right)} \phi\left(u_{n}-u\right) \frac{f\left(x, u_{n}\right)}{a(x)} d v_{g}+\tau_{n}\left\|\phi u_{n}\right\|+\tau_{n}\|\phi u\| \rightarrow 0 .
\end{aligned}
$$


We derive (3.16) from (3.21). Hence, (3.15) holds thanks to a covering argument. Since $\Sigma_{\delta}$ is a finite set, it follows that $\nabla_{g} u_{n}$ converges to $\nabla_{g} u$ almost everywhere in $M$. This immediately implies, up to a subsequence,

$$
\left|\nabla_{g} u_{n}\right|^{N-2} \nabla_{g} u_{n} \rightarrow\left|\nabla_{g} u\right|^{N-2} \nabla u \quad \text { in }\left(L^{N-1}\left(\mathbb{B}_{R}(0)\right)\right)^{N}
$$

for all $R>0$. For any fixed $\varphi \in C_{0}^{\infty}(M)$, there exists some $R_{0}>0$ such that the support of $\varphi$ is contained in the ball $\mathbb{B}_{R_{0}}(O)$. Hence,

$$
\lim _{n \rightarrow \infty} \int_{M}\left(\left|\nabla_{g} u_{n}\right|^{N-2} \nabla_{g} u_{n}-\left|\nabla_{g} u\right|^{N-2} \nabla_{g} u\right) \varphi d v_{g} \rightarrow 0
$$

This equality holds for all $\varphi \in L^{\frac{N}{N-1}}(M)$, thanks to the density of $C_{0}^{\infty}(M)$ in $L^{\frac{N}{N-1}}(M)$. Hence, we obtain

$$
\left|\nabla_{g} u_{n}\right|^{N-2} \nabla_{g} u_{n} \rightarrow\left|\nabla_{g} u\right|^{N-2} \nabla_{g} u \quad \text { in }\left(L^{\frac{N}{N-1}}(M)\right)^{N},
$$

we obtain by combining (3.6) and (3.22),

$$
\left\langle I^{\prime}(u), \varphi\right\rangle=0, \quad \forall \varphi \in C_{0}^{\infty}(M) .
$$

Since $C_{0}^{\infty}(M)$ is dense in $E$, the above equation implies that $u$ is a weak solution of $\left(P_{a}\right)$. This completes the proof of the lemma.

\section{The ground state solution}

Proof of Theorem 1.1 By the process of proof in Lemma 3.4, we see that the Cerami sequence $\left\{u_{n}\right\}$ is bounded in $E$ and its weak limit $u$ is a critical point of the functional $I$.

We will show that $u$ is nonzero. If $u \equiv 0$, since $F(x, 0)=0$ for all $x \in \mathbb{R}^{N}$, from Lemma 3.4, we have

$$
\lim _{n \rightarrow \infty}\left\|u_{n}\right\|_{E}^{N}=N c>0
$$

Lemma 3.3 implies $0<c<\frac{1}{N}\left(\left(1-\frac{1}{s}\right) \frac{\alpha_{N}}{\alpha_{0}}\right)^{N-1}$, so there exist some $\epsilon_{0}>0$ and $n_{*}>0$ such that $\left\|u_{n}\right\|_{E}^{N} \leq\left(\left(1-\frac{1}{s}\right) \frac{\alpha_{N}}{\alpha_{0}}-\epsilon_{0}\right)^{N-1}$ for all $n>n_{*}$. Choose $q>1$ sufficiently close to 1 such that $q \alpha_{0}\left\|u_{n}\right\|_{E}^{\frac{N}{N-1}} \leq\left(1-\frac{1}{s}\right) \alpha_{N}-\epsilon_{0} \alpha_{0} / 2$ for all $n>n_{*}$. By $\left(f_{1}\right)$,

$$
f\left(x, u_{n}\right) \leq c_{1}\left|u_{n}\right|^{N}+c_{2}\left|u_{n}\right| \Phi\left(\alpha_{0}\left|u_{n}\right|^{N-1}\right)
$$

thus

$$
\begin{aligned}
& \int_{M} \frac{\left|f\left(x, u_{n}\right) u_{n}\right|}{a(x)} d v_{g} \\
& \quad \leq c_{1} \int_{M} \frac{\left|u_{n}\right|^{N}}{a(x)} d v_{g}+c_{2} \int_{M} \frac{\left|u_{n}\right| \Phi\left(\alpha_{0}\left|u_{n}\right|^{\frac{N}{N-1}}\right)}{a(x)} d v_{g}
\end{aligned}
$$




$$
\begin{aligned}
& \leq c_{1} \int_{M} \frac{\left|u_{n}\right|^{N}}{a(x)} d v_{g}+c_{2}\left(\int_{M} \frac{\Phi\left(q \alpha_{0}\left|u_{n}\right|^{N-1}\right)}{a(x)} d v_{g}\right)^{\frac{1}{q}}\left(\int_{M} \frac{\left|u_{n}\right|^{q^{\prime}}}{a(x)} d v_{g}\right)^{\frac{1}{q^{\prime}}} \\
& \leq c_{1} \int_{M} \frac{\left|u_{n}\right|^{N}}{a(x)} d v_{g}+C\left(\int_{M} \frac{\left|u_{n}\right|^{q^{\prime}}}{a(x)} d v_{g}\right)^{\frac{1}{q^{\prime}}} \rightarrow 0
\end{aligned}
$$

here we used Lemma 2.2 in the last step. From $I^{\prime}\left(u_{n}\right) u_{n} \rightarrow 0$, we have

$$
\lim _{n \rightarrow \infty}\left\|u_{n}\right\|_{E}^{N}=0,
$$

which is a contradiction with (4.1), so $u$ is nonzero.

Now, we will show the existence of positive ground state solution for $\left(P_{a}\right)$. Setting

$$
m=\inf _{u \in \Lambda} I(u), \quad \Lambda:=\left\{u \in E \backslash\{0\}: I^{\prime}(u) u=0\right\} .
$$

Let $c$ be the mountain-pass level, obviously $m \leq c$.

On the other hand, let $u \in \Lambda$, then $u>0$. In fact, denote $u^{-}:=\min \{u, 0\}$, from $I^{\prime}(u) u^{-}=0$, we have $\left\|u^{-}\right\|=0$, thus $u \geq 0$ on $M$, from $I(u)=c>0$, we know $u$ is positive on $M$. Define $h:(0,+\infty) \rightarrow \mathbb{R}$ by $h(t)=I(t u)$. We see that $h$ is differentiable and

$$
h^{\prime}(t)=I^{\prime}(t u) u=t^{N-1}\|u\|^{N}-\int_{M} \frac{f(x, t u) u}{a(x)} d v_{g}, \quad \forall t>0 .
$$

From $I^{\prime}(u) u=0$, we get

$$
h^{\prime}(t)=I^{\prime}(t u) u-t^{N-1} I^{\prime}(u) u,
$$

so

$$
h^{\prime}(t)=t^{N-1} \int_{M}\left(\frac{f(x, u)}{u^{N-1}}-\frac{f(x, t u)}{(t u)^{N-1}}\right) \frac{u^{N}}{a(x)} d v_{g}, \quad \forall t>0 .
$$

By $\left(f_{6}\right)$ and $u>0$, we conclude that $h^{\prime}(t)>0$ for $0<t<1$ and $h^{\prime}(t)<0$ for $t>1$, since $h^{\prime}(1)=$ 0 , thus,

$$
I(u)=\max _{t \geq 0} I(t u)
$$

Now, define $\gamma:[0,1] \rightarrow E, \gamma(t)=t t_{0} u$, where $t_{0}$ is a real number which satisfies $I\left(t_{0} u\right)<0$, we have $\gamma \in \Gamma$, and therefore

$$
c \leq \max _{t \in[0,1]} I(\gamma(t)) \leq \max _{t \geq 0} I(t u)=I(u) .
$$

Since $u \in \Lambda$ is arbitrary, we have $c \leq m$, thus $c=m$. This completes the proof of Theorem 1.1.

\section{The nontrivial solution of perturbation problem}

In this section, we consider the following perturbation problem:

$$
-\operatorname{div}_{g}\left(\left|\nabla_{g} u\right|^{N-2} \nabla_{g} u\right)+V(x)|u|^{N-2} u=\frac{f(x, u)}{a(x)}+\epsilon h(x) \quad \text { in } M
$$

where $\epsilon>0, h \in E^{*}, h \geq 0$ and $h \not \equiv 0$. 
We say that $u \in E$ is a weak solution of problem $(P)$ if for all $\phi \in E$,

$$
\int_{M}\left(\left|\nabla_{g} u\right|^{N-2} \nabla_{g} u \nabla \phi+V(x)|u|^{N-2} u \phi\right) d v_{g}-\int_{M} \frac{f(x, u)}{a(x)} \phi d v_{g}-\epsilon \int_{\mathbb{R}^{N}} h(x) \phi d v_{g}=0 .
$$

Define the functional $I_{\epsilon}: E \rightarrow \mathbb{R}$ by

$$
I_{\epsilon}(u)=\frac{1}{N}\|u\|_{E}^{N}-\int_{\mathbb{R}^{N}} \frac{F(x, u)}{a(x)} d v_{g}-\epsilon \int_{M} h(x) u d v_{g},
$$

where $F(x, t)=\int_{0}^{t} f(x, s) d s . I$ is well defined and $I \in C^{1}(E, \mathbb{R})$ thanks to the singular Trudinger-Moser inequality. A straightforward calculation shows that

$$
\begin{aligned}
\left\langle I^{\prime}(u), \phi\right\rangle= & \int_{M}\left(\left|\nabla_{g} u\right|^{N-2} \nabla_{g} u \nabla_{g} \phi+V(x)|u|^{N-2} u \phi\right) d v_{g} \\
& -\int_{M} \frac{f(x, u)}{a(x)} \phi d v_{g}-\epsilon \int_{M} h(x) \phi d v_{g},
\end{aligned}
$$

for all $u, \phi \in E$, hence, a critical point of (5.2) is a weak solution of $(P)$.

Next, we will prove Theorem 1.3, one solution is obtained by using the mountain-pass theorem without Cerami condition.

Proof of Theorem 1.3 As in previous sections, it is similar to the first part of Theorem 1.1, $I_{\epsilon}$ satisfies the geometry condition of the mountain-pass theorem; i.e., $I_{\epsilon} \in C^{1}(E, \mathbb{R})$, there exists $\epsilon_{1}>0$ such that when $0<\epsilon<\epsilon_{1}, I_{\epsilon}(u) \geq \delta_{\epsilon}>0$ for $\|u\|_{E}=r_{\epsilon} ; I_{\epsilon}(e)<0$ for some $e \in E$ with $\|e\|_{E}>r_{\epsilon}$, where $r_{\epsilon}$ can be further chosen such that $r_{\epsilon} \rightarrow 0$ as $\epsilon \rightarrow 0$. Then, by the mountain-pass theorem without the Cerami condition, we get a $(C)_{c}$ sequence $\left\{u_{n}\right\} \subset E$; i.e.,

$$
I_{\epsilon}\left(u_{n}\right) \rightarrow c_{\epsilon} \quad \text { and } \quad\left(1+\left\|u_{n}\right\|_{E}\right)\left\|I_{\epsilon}^{\prime}\left(u_{n}\right)\right\|_{E^{*}} \rightarrow 0 \quad \text { as } n \rightarrow \infty
$$

where

$$
c_{\epsilon}=\inf _{\gamma \in \Gamma} \max _{t \in[0,1]} I_{\epsilon}(\gamma(t)) \geq \delta_{\epsilon}
$$

is the min-max value of $I_{\epsilon}$. By a similar argument to Lemma 3.4 to $I_{\epsilon}$, there exists a subsequence of $\left\{u_{n}\right\}$ that is bounded and its weak limit $u$ is a critical point of the functional $I_{\epsilon}$. This completes the proof of Theorem 1.3.

\section{Acknowledgements}

The authors are very grateful to the editorial board and the reviewers, whose comments improved the quality of the paper

\section{Funding}

This work was supported by NNSF of China (11471170, 11790271), Guangdong Basic and Applied basic Research Foundation (2020A1515011019), Innovation and development project of Guangzhou University, and Nankai Zhide Foundation.

Availability of data and materials

Not applicable. 
Authors' contributions

This entire work has been completed by the authors. The authors read and approved the final version of the manuscript.

\section{Author details}

${ }^{1}$ School of Mathematics and Information Science, Guangzhou University, Guangzhou, P.R. China. ${ }^{2}$ School of

Mathematical Sciences, Nankai University, Tianjin, P.R. China.

\section{Publisher's Note}

Springer Nature remains neutral with regard to jurisdictional claims in published maps and institutional affiliations.

Received: 23 May 2019 Accepted: 6 March 2020 Published online: 17 March 2020

\section{References}

1. Brézis, H.: Elliptic equations with limiting Sobolev exponents. Commun. Pure Appl. Math. 39, 517-539 (1986)

2. Brézis, H., Nirenberg, L.: Positive solutions of nonlinear elliptic equations involving critical Sobolev exponents. Commun. Pure Appl. Math. 36, 437-477 (1983)

3. Bartsch, T., Willem, M.: On an elliptic equation with concave and convex nonlinearities. Proc. Am. Math. Soc. 123, 3555-3561 (1995)

4. Capozzi, A., Fortunato, D., Palmieri, G.: An existence result for nonlinear elliptic problems involving critical Sobolev exponent. Ann. Inst. Henri Poincaré, Anal. Non Linéaire 2, $463-470$ (1985)

5. Garcia, A., Alonso, P.: Existence and non-uniqueness for the $p$-Laplacian. Commun. Partial Differ. Equ. 12, 1389-1430 (1987)

6. Adimurthi, A.: Existence of positive solutions of the semilinear Dirichlet problem with critical growth for the N-Laplacian. Ann. Sc. Norm. Super. Pisa XVII, 393-413 (1990)

7. Adimurthi, A., Yadava, S.: Multiplicity results for semilinear elliptic equations in a bounded domain of $\mathbb{R}^{2}$ involving critical exponent. Ann. Sc. Norm. Super. Pisa XVII, 481-504 (1990)

8. de Figueiredo, D.G., do Ó, J.M., Ruf, B.: On an inequality by N. Trudinger and J. Moser and related elliptic equations. Commun. Pure Appl. Math. IV, 135-152 (2002)

9. de Figueiredo, D.G., Miyagaki, O.H., Ruf, B.: Elliptic equations in $\mathbb{R}^{2}$ with nonlinearities in the critical growth range. Calc. Var. Partial Differ. Equ. 3, 139-153 (1995)

10. do Ó, J.M.: Semilinear Dirichlet problems for the $N$-Laplacian in $\mathbb{R}^{N}$ with nonlinearities in the critical growth range. J. Differ. Equ. 9, 967-979 (1996)

11. Panda, R.: On semilinear Neumann problems with critical growth for the N-Laplacian. Nonlinear Anal. 26, 1347-1366 (1996)

12. Lam, N., Lu, G.: Elliptic equations and systems with subcritical and critical exponential growth without the Ambrosetti-Rabinowitz conditions. J. Geom. Anal. 24, 118-143 (2014)

13. Trudinger, N.S.: On embeddings into Orlicz spaces and some applications. J. Math. Mech. 17, 473-484 (1967)

14. Moser, J: A sharp form of an inequality by N. Trudinger. Indiana Univ. Math. J. 20, 1077-1091 (1971)

15. Kryszewski, W., Szulkin, A.: Generalized linking theorem with an application to semilinear Schrödinger equation. Adv. Differ. Equ. 3, 441-472 (1998)

16. Alama, S., Li, Y.: Existence of solutions for semilinear elliptic equations with indefinite linear part. J. Differ. Equ. 96 89-115 (1992)

17. Ding, W., Ni, W.: On the existence of positive entire solutions of a semilinear elliptic equation. Arch. Ration. Mech. Anal. 31, 283-308 (1986)

18. Jeanjean, L.: Solutions in spectral gaps for a nonlinear equation of Schrödinger type. J. Differ. Equ. 112, 53-80 (1994)

19. Cao, D.: Nontrivial solution of semilinear elliptic equations with critical exponent in $\mathbb{R}^{2}$. Commun. Partial Differ. Equ. 17, 407-435 (1992)

20. Panda, R.: Nontrivial solution of a quasilinear elliptic equation with critical growth in $\mathbb{R}^{N}$. Proc. Indian Acad. Sci. Math. Sci. 105, 425-444 (1995)

21. do Ó, J.M.: N-Laplacian equations in $\mathbb{R}^{N}$ with critical growth. Abstr. Appl. Anal. 2, 301-315 (1997)

22. Alves, C.O., Figueiredo, G.M.: On multiplicity and concentration of positive solutions for a class of quasilinear problems with critical exponential growth in $\mathbb{R}^{N}$. J. Differ. Equ. 246, 1288-1311 (2009)

23. do Ó, J.M., Medeiros, E., Severo, U.: On a quasilinear nonhomogeneous elliptic equation with critical growth in $\mathbb{R}^{N}$. J. Differ. Equ. 246, 1363-1386 (2009)

24. Aubin, T.: Sur la function exponentielle. C. R. Acad. Sci. Paris, Ser. A 270, 1514-1516 (1970)

25. Cherrier, P.: Une inégalité de Sobolev sur les variétés Riemanniennes. Bull. Sci. Math. 103, 353-374 (1979)

26. Cherrier, P.: Cas d'exception du théorème d'inclusion de Sobolev sur les variétés Riemanniennes et applications. Bull. Sci. Math. 105, 235-288 (1981)

27. Fontana, L.: Sharp borderline Sobolev inequalities on compact Riemannian manifolds. Comment. Math. Helv. 68, 415-454 (1993)

28. Ding, W., Jost, J., Li, J., Wang, G.: The differential equation $\Delta u=8 \pi-8 \pi$ he $e^{u}$ on a compact Riemann surface. Asian J. Math. 1, 230-248 (1997)

29. Li, Y: Moser-Trudinger inequality on compact Riemannian manifolds of dimension two. J. Partial Differ. Equ. 14 163-192 (2001)

30. Li, Y.: The extremal functions for Moser-Trudinger inequality on compact Riemannian manifolds. Sci. China Ser. A 48, 618-648 (2005)

31. Yang, Y.: A sharp form of Moser-Trudinger inequality on compact Riemannian surface. Trans. Am. Math. Soc. 359 5761-5776 (2007)

32. Yang, Y: A sharp form of trace Moser-Trudinger inequality on compact Riemannian surface with boundary. Math. Z 255, 373-392 (2007) 
33. Yang, Y.: Trudinger-Moser inequalities on complete noncompact Riemannian manifolds. J. Funct. Anal. 263, 1894-1938 (2012)

34. Yang, Q., Su, D., Kong, Y.: Sharp Moser-Trudinger inequalities on Riemannian manifolds with negative curvature. Ann. Mat. Pura Appl. (4) 195, 459-471 (2016)

35. Kristály, A.: New geometric aspects of Moser-Trudinger inequalities on Riemannian manifolds: the non-compact case. J. Funct. Anal. 276, 2359-2396 (2019)

36. Adimurthi, Yang, Y.: An interpolation of Hardy inequality and Trudinger-Moser inequality in $\mathbb{R}^{N}$ and its applications. Int. Math. Res. Not. 13, 2394-2426 (2010)

37. Yang, Y.: Existence of positive solutions to quasilinear elliptic equations with exponential growth in the whole Euclidean space. J. Funct. Anal. 262, 1679-1704 (2012)

38. Lam, N., Lu, G.: Existence and multiplicity of solutions to equations of $n$-Laplacian type with critical exponential growth in $\mathbb{R}^{N}$. J. Funct. Anal. 262, 1132-1165 (2012)

39. Cerami, G.: An existence criterion for the critical points on unbounded manifolds. Ist. Lomb., Accad. Sci. Lett., Rend. A 112, 332-336 (1978) (Italian)

40. Cerami, G.: On the existence of eigenvalues for a nonlinear boundary value problem. Ann. Mat. Pura Appl. 124(4), 161-179 (1980) (Italian)

\section{Submit your manuscript to a SpringerOpen ${ }^{0}$} journal and benefit from:

- Convenient online submission

- Rigorous peer review

- Open access: articles freely available online

- High visibility within the field

- Retaining the copyright to your article

Submit your next manuscript at $\boldsymbol{s p r i n g e r o p e n . c o m ~}$ 